\title{
Liquid-Infused Membranes with Oil-in-Water Emulsions
}

\author{
Hanieh Bazyar, ${ }^{\dagger, \ddagger}$ Noor van de Beek, ${ }^{\dagger}$ and Rob G. H. Lammertink* ${ }^{* \dagger}$ \\ ${ }^{\dagger}$ Soft Matter, Fluidics and Interfaces, MESA+Institute for Nanotechnology, University of Twente, P.O. Box 217, 7500 AE Enschede, \\ The Netherlands \\ ${ }^{*}$ Wetsus, European Centre of Excellence for Sustainable Water Technology, P.O. Box 1113, 8900 CC Leeuwarden, The Netherlands
}

Supporting Information

ABSTRACT: Liquid-infused membranes have been introduced to membrane technology recently. The infusion liquid can be expelled, opening the pore, in response to an immiscible feed liquid pressure. In the open state, the pore wall is still covered with the infusion liquid forming the so-called liquid-lined pores. Liquid lining is expected to give anti-fouling properties to these membranes. The pressureresponsive pores can be used for efficient sorting of fluids from a mixture based on interfacial tension. For example, in a two-phase mixture of immiscible liquids, the required liquid entry pressure is different for the constituent liquids. Here, we

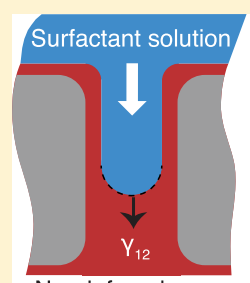

Non-infused pore

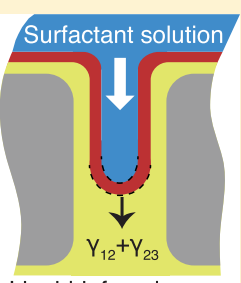

Liquid-infused pore investigate the capability of liquid-infused membranes for selective permeation of the dispersed phase, that is, oil from an oil-in-water $(\mathrm{O} / \mathrm{W})$ emulsion. The separation experiments are conducted in a dead-end pressure-controlled filtration cell using liquid-infused and non-infused membranes. In order to permeate the dispersed phase, oil droplets should come in contact with the membrane surface which is accomplished here by gravity-driven creaming. Our results reveal that by setting the feed pressure between the entry pressure of oil and that of the surfactant solution, oil can be successfully permeated. For high concentrations of surfactants, water also permeated partly. The amount of water permeated through liquid-infused membranes is lower than that through non-infused membranes, caused by the corresponding interfacial tensions. The results suggest that the presence of the infusion liquid in the membrane gives rise to the formation of three-phase interfaces in the pore, namely, the interface between surfactant solution-oil $\left(\gamma_{12}\right)$ and that between oil-infusion liquid $\left(\gamma_{23}\right)$. Based on the interfacial energy contributions, the additional interface between oil and the infusion liquid gives rise to an increase in the liquid entry pressure for the surfactant solution based on the combined interfacial tension $\left(\gamma_{12}+\gamma_{23}\right)$ leading to less water permeation.

\section{INTRODUCTION}

The rapid industrial growth in the field of oil and gas has led to the production of large amounts of oily waste water. ${ }^{1}$ Oilcontaining waste water is also produced in industries related to edible oil and detergent processing, textile (scouring water), pharmacy, and metallurgy. ${ }^{1,2}$ These waste water streams can be either reused or discharged into coastal water or municipal sewage systems. Purification before discharge is of evident importance. Regulations require that the maximum total oil and grease concentration in discharge waters should be less than $10 \mathrm{mg} / \mathrm{L}^{3}$

Several methods to remove oil from waste water include conventional physical and chemical methods. Adsorption using activated carbon or resins, sand filters, and evaporation of water are examples of physical treatments. Chemical treatment methods include oxidation, electrochemical conversion, and photocatalytic degradation. High cost, using toxic compounds, large space for installation, and generation of secondary pollutants are severe drawbacks of the aforementioned conventional methods. ${ }^{4}$ Membrane separation methods involving polymeric as well as ceramic membranes have been recently introduced as an efficient technique to treat oily waste water. ${ }^{4,5}$ The advantages of membrane processes include lower capital cost, facile fabrication processes, and no necessity for chemical additives while generating permeates of acceptable quality. ${ }^{6}$ The porous structure of the membrane can act as a conventional coalescer. Permeation of the emulsion through the membrane pores promotes coalescence of micron and submicron oil droplets resulting in destabilization of the emulsion. ${ }^{7}$ The coalesced large oil droplets can be further separated from the aqueous phase by the use of gravity for instance. ${ }^{8}$

The methods for oily waste water treatment based on membrane separation processes rely on reverse osmosis, ${ }^{9}$ microfiltration (MF), ${ }^{10,11}$ membrane distillation, ${ }^{12}$ and ultrafiltration (UF). ${ }^{6,13,14}$ UF is considered to be one of the most effective treatment techniques for oil-water separation.,15 Surface-modified polyethersulfone and polysulfone UF membranes showed oil retention of 100 and $90 \%$, respectively, reducing the oil concentration below $10 \mathrm{mg} / \mathrm{L}$ in the aqueous permeate. ${ }^{6,16}$ Ceramic MF membranes exhibited $98.8 \%$ oil rejection efficiency, which yielded permeate streams with environmentally acceptable oil concentration. ${ }^{5}$

Electrospun fiber membranes were also used for the separation of $\mathrm{O} / \mathrm{W}$ emulsions. ${ }^{17-20}$ The membranes were tested in both dead-end and cross flow filtration modes

Received: April 10, 2019

Revised: June 19, 2019

Published: June 26, 2019 
showing around $93 \%$ oil rejection. ${ }^{17}$ It was shown that further surface modification of electrospun fiber membranes can lead to hierarchal superhydrophilic structures with underwater superoleophobicity. Surface modification methods include coating by graphene oxide, ${ }^{18}$ formation of nanoclusters on cross-linked membranes, ${ }^{19}$ and electrospraying of nanoparticles. $^{20}$ These membranes showed good separation efficiency (>98\%) with high water flux values.

Membranes with peculiar superwetting properties, that is, inair superamphiphilic, underwater superoleophobic, and underoil superhydrophobic, showed their potential in separation of both micrometer- and nanometer-size $\mathrm{O} / \mathrm{W}$ and water-in-oil (W/O) emulsion with separation efficiency of more than 99\%. ${ }^{21-24}$ These membranes are mainly hybrid/composite porous membranes with hierarchal micro-/nanostructural surface that are achieved by embedding nano-/microparticles to the membrane structure. ${ }^{25,26}$ Huang et al. showed that combining silica nanofibers and in situ polymerized fluorinated polybenzoxazine can be used to fabricate a superhydrophobic superoleophilic nanofibrous membrane. This membrane is further used for gravity-driven separation of oil from $\mathrm{W} / \mathrm{O}$ emulsions in which no external driving force is needed. High flux values with good antifouling properties and thermal stability were observed. ${ }^{27}$ Recently, Janus membranes, that is, membranes with asymmetric properties on each side, have been used for unidirectional oil-water separation. ${ }^{28,29}$ A hydrophilic-hydrophobic Janus membrane was used to separate oil from $\mathrm{O} / \mathrm{W}$ emulsion. The hydrophilic side was exposed to the emulsion, which facilitated the coalescence of oil droplets. The oil-filled superhydrophobic pores further prevented water permeation. $^{29}$

Materials with superwetting properties have found their applications in absorbents as well. "Smart sorbents" in which materials' adsorption properties can be controlled electrically, thermally, or magnetically are gaining popularity in $\mathrm{O} / \mathrm{W}$ separation field. ${ }^{30}$ Abdulhussein et al. newly developed a superhydrophobic, superoleophilic magnetic, porous material based on polydimethylsiloxane (PDMS) and steel wool (SW). The PDMS-modified SW can be magnetically guided and is capable of absorbing and separating oils and organic solvents from water. Gravity-driven oil-water separation results showed permeate flux as high as $\sim 32000 \mathrm{~L} / \mathrm{m}^{2} \mathrm{~h}$ with separation efficiency over $99 \%$. $^{31}$

Limited strength, durability, and unsatisfactory performance are the main drawbacks of membranes with superwetting/ antiwetting properties. ${ }^{21}$ Multiple-step fabrication procedure and in some cases requirement of special chemical or sophisticated techniques further limit wide-spread application of the aforementioned membranes. Additionally, in all these membrane processes, the continuous aqueous phase permeates through the membrane providing a concentrated retentate and purified water as permeate (Figure 1a). The consequence of this is that a large volume of continuous water phase has to permeate, with accompanying fouling challenges. A method to reduce the permeating volume is to permeate the dispersed phase instead of the continuous phase (Figure 1b). Permeation of the dispersed phase from an $\mathrm{O} / \mathrm{W}$ emulsion using a membrane requires two steps. First, the dispersed phase must come in contact with the membrane and likely coalesce on the surface. Second, the oil should permeate through the membrane with little or no water, requiring some degree of affinity between the oil and membrane phase. ${ }^{32}$
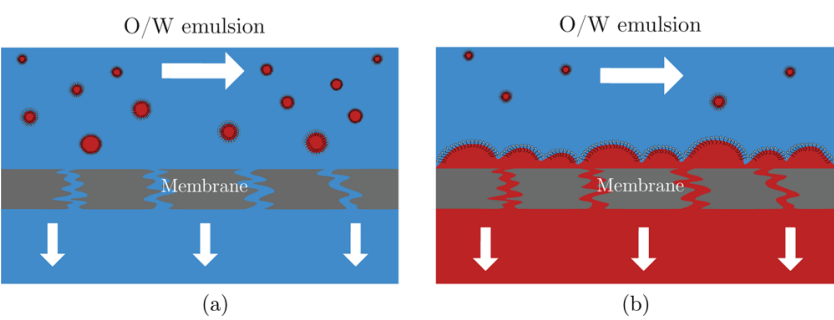

Figure 1. Membrane process for oil separation from $\mathrm{O} / \mathrm{W}$ emulsion by (a) permeation of the continuous water phase (blue) and (b) permeation of the dispersed oil phase (red).

Here, we report on the application of a novel type of membrane, that is a liquid-infused membrane, for selective permeation of the dispersed phase from an $\mathrm{O} / \mathrm{W}$ emulsions. An additional liquid phase that is present in the membrane concerns an alternative approach to oil-water separation using membranes. These membranes are inspired by a new class of functional surfaces, known as slippery liquid-infused porous surfaces (SLIPS). ${ }^{33}$ The capillary-stabilized liquid in the nano-/microstructured surface forms a smooth lubrication layer on the surface responsible for its low water contact angle hysteresis and low-fouling properties. ${ }^{34-36}$ Other important features of these surfaces include self-healing by capillary wicking, repelling a variety of liquids, and anti-biofouling. ${ }^{33,34}$ Fabrication of SLIPS often requires multistep processing, high temperatures, and drying mainly to render the substrate hydrophobic and match the chemistry between solid and infusion liquid. ${ }^{37,38}$ Porous polymeric membranes with low surface energies may provide effective alternatives. These membranes are typically prepared from fluorinated polymers, for example polyvinylidene fluoride (PVDF) and polytetrafluoroethylene. ${ }^{36,39,40}$ Liquid infusion has been proved to be a good technique to improve the water sliding properties of surfaces. Hu et al. demonstrated that infusion of silicone oil into antismudge polyurethane-based coatings (SINP-GLIDE) can decrease the sliding angle of water and various organic liquids. Water sliding properties did not degrade after subjecting to violent simulated rain cycles. ${ }^{41}$ Recently, it was demonstrated that liquid-filled pores can form a reconfigurable gate, which allows liquid permeation based on interfacial tension (IFT). This gating mechanism gives liquid-infused membranes the capability of multiphase transport without clogging. ${ }^{36,42}$ In for example a two-phase mixture of immiscible fluids, like $\mathrm{O} / \mathrm{W}$ emulsions, the selective permeation of one fluid and retention of the other one can be achieved by setting the feed pressure between the entry pressure of the permeating fluid and that of the retained fluid. The entry pressure is set by the Laplace pressure ${ }^{43}$

$$
\Delta P=\frac{2 \gamma\left|\cos \theta_{\mathrm{E}}\right|}{r}
$$

where $r$ is the pore radius $(\mathrm{m}), \gamma$ is the IFT $(\mathrm{N} / \mathrm{m})$ between permeating fluid and liquid, and $\theta_{\mathrm{E}}$ is the advancing contact angle (larger than $90^{\circ}$ ) of the permeating fluid with respect to the pore wall.

The gating mechanism of liquid-infused membranes was previously explored via gas-liquid displacement porometry. ${ }^{40}$ The liquid film thickness on the pore wall (liquid-lining) was estimated experimentally and theoretically. Liquid-liquid displacement porometry measurements showed the presence of liquid-lined pores even after pushing pure water through 


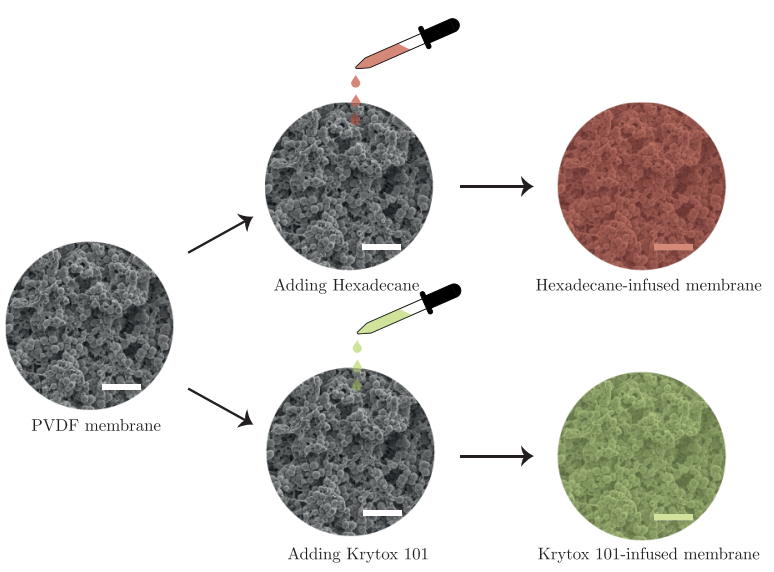

(a)
Liquid-infused membrane

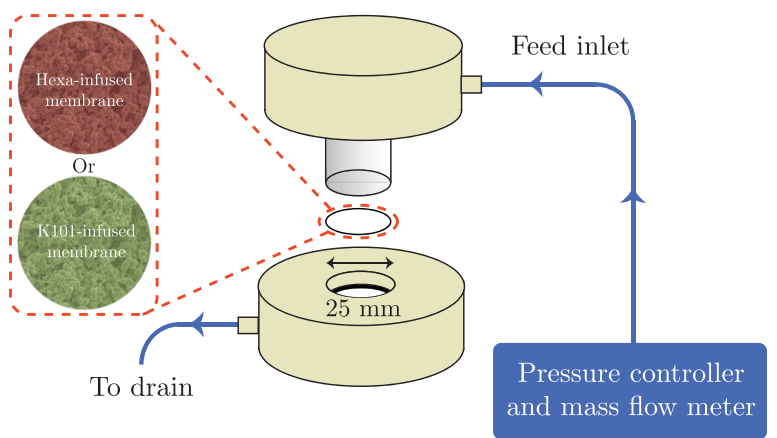

(b)

Figure 2. (a) SEM image of the membrane (scale bar is $10 \mu \mathrm{m}$ ) with schematic illustration of fabrication of liquid-infused membranes. (b) Schematic illustration of the LEP measurement setup.

liquid-infused membranes at high flux values. ${ }^{44}$ The observed displacement mechanism, visualized using microfluidics, revealed liquid-lining and preferential flow pathways for water transport. The presence of an additional liquid layer on the pore wall prevents direct contact between the permeating fluid and the solid material of the membrane giving rise to the potential antifouling properties. ${ }^{36}$ The antifouling properties of liquid-infused membranes as well as pressure-responsive pores for selective fluid transport are attractive for separation applications. The good performance of these membranes in efficient sorting of a three-phase airwater-oil mixture ${ }^{36}$ makes them a potential candidate for oily waste water treatment.

In this work, the performance of liquid-infused membranes for oil permeation from $\mathrm{O} / \mathrm{W}$ emulsions is investigated and compared to non-infused counterparts (dry membrane). The underlying mechanism for the improved performance of the liquid-infused membranes for selective oil permeation is further explored in detail. This work shows the potential application of liquid-infused membranes to treat oily waste water.

\section{EXPERIMENTAL SECTION}

Materials. PVDF (Solef 6020/1001) was received from Solvay Solexis, France. 1-Methyl-2-pyrrolidinone (NMP) (99\% extra pure) was purchased from Acros Organics, The Netherlands. Ethanol $(100 \% \mathrm{v} / \mathrm{v})$ technical grade was supplied from Boom Chemical Company, The Netherlands. Krytox GPL oil 101 was purchased from MAVOM Chemical Industry, The Netherlands. Hexadecane (reagent plus 99\%), 2-propanol (anhydrous, 99.5\%), sudan IV (dye content $\geq 80 \%$ ), perylene fluorescent dye (sublimed grade $\geq 99.5 \%$ ), sodium dodecyl sulfate (SDS) (ACS reagent, $\geq 99.0 \%$ ), and cetyltrimethylammonium bromide (CTAB) $(\geq 99 \%)$.

Fabrication of Membrane and Dense PVDF Film. The polymer solution was prepared by mixing 15 wt \% PVDF in NMP with a mechanical stirrer overnight at $80^{\circ} \mathrm{C}$. The solution was cast on a glass plate using a casting knife at an initial thickness of $500 \mu \mathrm{m}$. The cast membrane was immediately submerged in water/NMP (25:75 vol \%) as the coagulation bath for $60 \mathrm{~min}$. The remaining NMP was removed from the membrane by subsequently keeping it in ethanol for another $60 \mathrm{~min}$. The cast membrane was then attached to a piece of paper using paper clips to prevent curling and left to dry in a fume hood $(60 \mathrm{~min})$ before placing it in a $30^{\circ} \mathrm{C}$ vacuum oven overnight.

Dense PVDF film was made by casting the polymer dope solution on a glass plate using the same procedure as described for membrane fabrication. The cast polymer solution was placed in a box and dried with a flow of nitrogen for 2 days.

Membrane Characterization. The pore size distribution of the membrane was characterized using a capillary flow porometer (POROLUX 1000) (see Supporting Information, Figure S2). The morphology of the top, bottom, and cross section of the membranes were observed using a scanning electron microscopy (SEM) system (JEOL $5600 \mathrm{LV}$ ) (see Supporting Information, Figure S1). The surface wetting properties of the fabricated membranes were investigated using a contact angle goniometer (DataPhysics OCA20) (see Table 4).

Fabrication of Liquid-Infused Membranes. Liquid-infused membranes were prepared by adding an over coat layer $(15.5 \mu \mathrm{L}$ $\mathrm{cm}^{-2}$ ) of perfluoropolyether (PFPE) oil, that is, Krytox GPL 101 oil (K101), on the membranes using a micropipette. In order to have a high affinity between the infusion liquid and the PVDF membrane, a fluorinated oil, that is, K101, was used as the infusion liquid. This is an important criteria for design and fabrication of liquid-infused surfaces. ${ }^{33}$ Hexadecane-infused membranes were prepared using the same procedure by adding hexadecane to the membrane. Both oils spontaneously infiltrated the pores via capillary wicking (Figure 2a).

Preparation of Surfactant Solutions. Two different surfactants (anionic SDS and cationic CTAB) were used to prepare the surfactant solutions (see Table 1). Four solutions have been prepared by

Table 1. Overview of the Used Surfactants and the Corresponding cmc Values Measured Experimentally (See Supporting Information Figures S11 and S15)

\begin{tabular}{lll}
\multicolumn{1}{c}{ surfactant } & charge & \multicolumn{1}{c}{$\mathrm{cmc}(\mathrm{mM})$} \\
sodium dodecyl sulfate (SDS) & anionic & $8.1\left(\right.$ at $\left.20-25{ }^{\circ} \mathrm{C}\right)$ \\
cetyltrimethylammonium bromide & cationic & $0.92\left(\right.$ at $20-25{ }^{\circ} \mathrm{C}$ ) \\
$(\mathrm{CTAB})$ & &
\end{tabular}

dissolving 10 and $50 \%$ critical micelle concentration $(\mathrm{cmc})$ of each surfactant in pure water (Milli-Q grade). A period of more than $12 \mathrm{~h}$ was given for all surfactants to completely dissolve in water.

Measurement of Liquid Entry Pressure. The liquid entry pressures (LEP) of all the aforementioned surfactant solutions (see Table 1) through K101-infused and hexadecane-infused membranes were measured. The measurement was performed by increasing the pressure step-wise, while measuring the permeate flux using a Bronkhorst pressure controller (IN-PRESS P-502CI) and a mass flow meter (mini CORI-FLOW M14), which were connected using a control valve (COMBI-FLOW Mass flow C5I). Depending on the surfactant type and the infusion liquid, pressure steps of 22 mbar or 5.5 mbar were used. The small steps $(5.5 \mathrm{mbar}$, which is the accuracy 
of the pressure controller) were considered for surfactant solutions with low IFT values with hexadecane, that is, CTAB 10 and 50 (see Figure 4a). At each step, the pressure was kept constant for $200 \mathrm{~s}$. The pressure at which the solution started permeating through the membrane and a stable flux was measured was defined as the LEP. The schematic illustration of the setup is shown in Figure $2 b$.

Measurements of IFT. The IFT values between different surfactant solutions and oils, Krytox 101 and hexadecane, were measured using a contact angle goniometer (DataPhysics OCA20). The pendant drop method was used for this measurements based on the drop shape analysis (see Figure $4 \mathrm{a}$ and Supporting Information for more information on the procedure and Table S2).

Preparation and Characterization of Emulsions. The $\mathrm{O} / \mathrm{W}$ emulsions have been prepared by dispersing 5 wt \% of hexadecane in the surfactant solution. A mechanical dispersing instrument (IKA ULTRA-TURRAX T18 digital) was used for emulsification. The emulsion was prepared by slowly adding the oil using a long needle [stainless steel 304 syringe needle, noncoding point (Z117056-1EA)] and a syringe to a 11 Schott DURAN glass bottle containing the surfactant solution. The mixing was done at $14000 \mathrm{rpm}$ for $15 \mathrm{~min}$. All the used devices including dispensing instrument, syringe, and the needle were cleaned thoroughly with 2-propanol and rinsed with pure water (Milli-Q grade) before preparation of the new emulsions. In order to better distinguish between oil and water in oil permeation experiments, hexadecane was dyed red using sudan IV dye (see Supporting Information, Figure S3a). The observation of the emulsion was done using an inverted laser scanning confocal microscopy (LSCM) system (Al system, Nikon Corporation, Japan) with a $40 \times$ dry objective (Plan Fluor, 40× DIC M N2, numerical aperture $=0.75$, calibration $0.62 \mu \mathrm{m} / \mathrm{px}$ ). For this purpose, an oil-soluble fluorescent dye (perylene) was added to hexadecane (see Supporting Information Figure S3b,c for the picture and the 3D image of the prepared emulsion respectively). The droplet size distribution of the $\mathrm{O} / \mathrm{W}$ emulsion was measured using a dynamic light scattering system (Mastersizer 2000) (see Figure 5 and Supporting Information section "droplet size distribution of prepared emulsions").

Oil Permeation from the O/W Emulsion Experiments. Unlike normal filtration in which the continuous phase is permeating through the membrane, the challenge here is to permeate the dispersed phase while retaining the continuous phase. To achieve this, oil droplets should come in contact with the membrane, which was obtained by placing the filtration cell upside down. A schematic illustration of the setup together with the LSCM image of the emulsion made with $10 \%$ cmc of SDS is shown in Figure 3. To observe the fabricated emulsion in the cell, the liquid chamber of the cell has been made out of transparent polymethyl methacrylate.

Oil (hexadecane) permeability of the non-infused (dry) and K101infused membranes was measured based on liquid-liquid displacement (LLD) experimental results (see Supporting Information, section "LLD analysis of hexadecane through liquid-infused membrane"). In LLD experiments, pure hexadecane was pushed

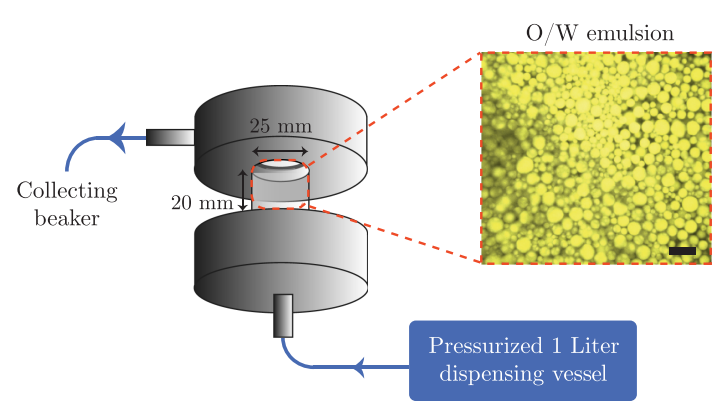

Figure 3. Schematic illustration of the dead-end filtration setup and LSCM image of the emulsion (scale bar is $25 \mu \mathrm{m}$ ). The dispensing vessel was filled with $500 \mathrm{~mL}$ of emulsion and pressurized with nitrogen gas. The pressure was controlled using a pressure regulator. through the membrane at increasing flux while measuring pressure. Permeability was measured according to Darcy's law (eq S1) based on the corresponding linear relation between oil flux and pressure.

\section{RESULTS AND DISCUSSION}

LEP and IFT Measurement Results. The IFT values between surfactant solutions and oils, Krytox 101 (K101) and hexadecane, were measured using the pendant drop method (see Supporting Information for more information on the procedure and Figures S11 and S15). The results up to 50\% of the corresponding $\mathrm{cmc}$ values are shown in Figure 4a. The experimental data points are fitted to the Szyszkowski equation, where the relation between IFT and surfactant concentration is defined as ${ }^{45}$

$$
\gamma=\gamma^{*}-b \gamma^{*} \ln \left(1+\frac{C}{a}\right)
$$

Here, $C$ is the surfactant concentration $\left(\mathrm{mol} / \mathrm{m}^{3}\right), a$ and $b$ are the fitting parameters, $\gamma$ is the IFT between oil and the surfactant solution $(\mathrm{N} / \mathrm{m})$, and $\gamma^{*}$ is the IFT value between pure water and pure oil $(\mathrm{N} / \mathrm{m}$ ) (see Supporting Information Table S2). Fitting was done using the least square fitting method in MATLAB. The fitting parameters $a$ and $b$ are summarized in Table 2 (see Supporting Information Figures S12 and S16 for the fitting results together with the corresponding 95\% confidence intervals bounds).

The LEP results of all the surfactant solutions through K101 and hexadecane-infused membranes are shown in Figure $4 \mathrm{~b}$. The LEP data points are fitted to the Laplace equation (eq 1) by considering complete non-wetting $\left(\theta_{\mathrm{E}}=180^{\circ}\right)$ and the Szyszkowski relation (eq 2) for the corresponding IFT value. The surfactant concentration in eq 2 is considered to be the concentration in the bulk solution, that is, $C_{0}$ leading to

$$
\Delta P=\frac{2 \gamma^{*}}{r}\left(1-b \ln \left(1+\frac{C_{0}}{a}\right)\right)
$$

Equation 3 relates the LEP of each surfactant solution to the surfactant concentration and radius of curvature $r$. The fitted values for the curvature radii (see Table 2) are smaller than the largest pore radius of the membrane, $1.92 \mu \mathrm{m}$, suggesting some pore-narrowing due to liquid-lining.

It is worth mentioning that as the surfactant solution is pushed through the membrane pore, surfactant can get adsorbed on the liquid-lined pore leading to a lower local concentration in the pore $\left(C_{\mathrm{P}}\right)$ compared to that of the bulk concentration $\left(C_{0}\right)$. In eq 3 , adsorption is considered in equilibrium with the bulk concentration $C_{0}$. By including surfactant adsorption on the liquid-lined pore, the surfactant concentration in the pore $\left(C_{\mathrm{P}}\right)$ should be used in eq 3. $C_{\mathrm{P}}$ values highly depend on pore radius $r$ (see Supporting Information section "surfactant adsorption on the liquid-lined pore in hexadecane-infused membranes" for more information on the relation between $C_{0}$ and $C_{\mathrm{p}}$ ). We observed that using $C_{0}$ matched with the experimental LEP. This suggests that the initial entry interface contains adsorbed surfactant in equilibrium with the bulk concentration, which is not significantly affected by any surfactant depletion due to adsorption.

In order to further choose the proper operating pressure for dead-end filtration, the LEP of pure hexadecane through dry and K101-infused membranes have been also measured. As expected, hexadecane wicks spontaneously through dry 


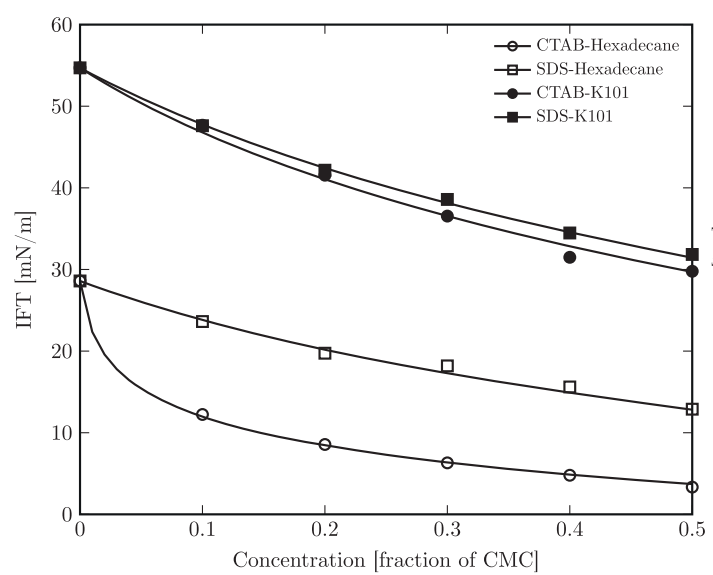

(a)

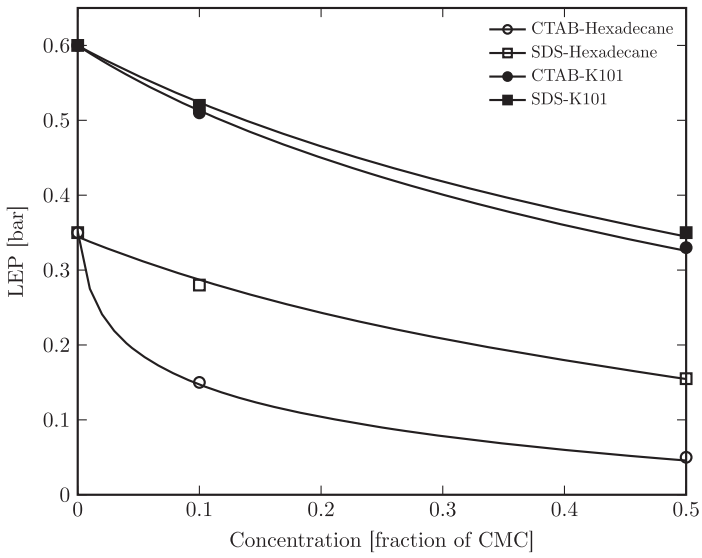

(b)

Figure 4. (a) IFT values between surfactant solutions, hexadecane, and K101 as a function of surfactant concentration [symbols are experiments, lines are fitted to the Szyszkowski equation (eq 2)]. (b) LEP values of surfactant solutions through K101- and hexadecane-infused membranes (symbols are experiments, lines are fitted to eq 3 ).

Table 2. Fitting Parameters of Fitted Experimental Data Points of IFT and LEP to the Szyszkowski Equation (Eq 2) and Laplace Equation by Considering Szyszkowski Relation for the IFT Value (Eq 3), Respectively [All the Values after \pm Are Half of the Confidence Interval Limits ((Upper CI - Lower CI)/2)]

\begin{tabular}{lcc} 
& \multicolumn{2}{c}{ fitting parameters } \\
\cline { 2 - 3 } surfactant solution-oil & $a\left(\mathrm{~mol} / \mathrm{m}^{3}\right)$ & $b(-)$ \\
CTAB-hexadecane & $4.02 \times 10^{-3} \pm 1.75 \times 10^{-3}$ & $1.83 \times 10^{-1} \pm 1.66 \times 10^{-2}$ \\
CTAB-K101 & $2.01 \times 10^{-1} \pm 4.93 \times 10^{-2}$ & $3.83 \times 10^{-1} \pm 5.13 \times 10^{-2}$ \\
SDS-hexadecane & $2.08 \pm 8.31 \times 10^{-1}$ & $5.11 \times 10^{-1} \pm 1.16 \times 10^{-1}$ \\
SDS-K101 & $2.24 \pm 2.64 \times 10^{-1}$ & $4.12 \times 10^{-1} \pm 2.81 \times 10^{-2}$
\end{tabular}

membrane, and therefore the LEP is zero. For a K101-infused membrane, a LEP for hexadecane of 0.08 bar was measured corresponding to an IFT of $8.7 \pm 0.08 \mathrm{mN} / \mathrm{m}$ (see Supporting Information Table S1).

Oil Permeation from the $\mathrm{O} / \mathrm{W}$ Emulsion. The comparison of the number-averaged droplet size distribution of all the prepared emulsions are shown in Figure 5 (see Supporting Information Figure S10 for the number- and volume-averaged droplet size distribution of each emulsion, separately).

One can calculate the creaming rate for each emulsion according to Stokes's law,

$$
u=\frac{2 a^{2}\left(\rho-\rho_{0}\right) g}{9 \mu}
$$

where $a$ is the droplet radius $(\mathrm{m}),\left(\rho-\rho_{0}\right)$ is the density difference between continuous and dispersed phases $\left(\mathrm{kg} / \mathrm{m}^{3}\right)$, $g$ is the gravitational acceleration $\left(\mathrm{m} / \mathrm{s}^{2}\right)$, and $\mu$ is the dynamic viscosity of the continuous phase ( $\mathrm{Pa} \mathrm{s}) .{ }^{46}$ For example, the creaming rate of the emulsion made with SDS10 is between 0.06 and $19.85 \mu \mathrm{m} / \mathrm{s}$, corresponding to the smallest and largest droplets, respectively. Once the droplets reach the membrane surface, they will coalesce and subsequently form a hexadecane oil layer (see Supporting Information Figure S6d) on the membrane surface. By applying a transmembrane pressure (TMP), which is higher than the LEP of hexadecane and lower than that of the surfactant solutions (see section LEP and IFT Measurement Results), oil can selectively permeate through the membrane. The applied TMP for all the experiments was smaller than 0.1 bar. Dry membranes as well as K101-infused membranes were tested for selective oil permeation using all

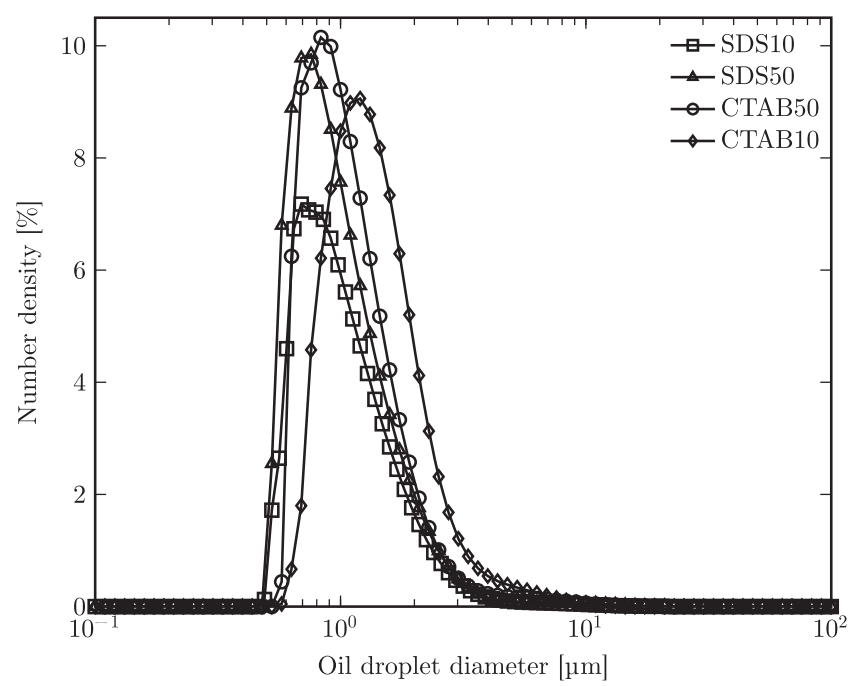

Figure 5. Comparison of the number density droplet size distribution of all the prepared hexadecane in water emulsions, with indicated surfactant concentration.

prepared $\mathrm{O} / \mathrm{W}$ emulsions. The performance of these two membranes were compared and the results are summarized in Table 3. The amount of water in each permeate was estimated from the corresponding collected permeate (see Supporting Information Figures S7-S9).

It is worth mentioning that in the dead-end filtration experiments the flux is mainly set by the concentration of the minor phase. As a result, oil permeation in dead-end filtration mode is a discontinuous process and limited by the amount of 
Table 3. Amount of Water Permeated through Dry and K101-Infused Membranes as a Function of Surfactant Concentration during Oil Permeation Experiments at TMP $\leq 0.1$ bar

\begin{tabular}{lcl} 
& \multicolumn{2}{c}{ fraction of water in the permeate } \\
\cline { 2 - 3 } emulsion & K101-infused & dry \\
SDS 10 & 0 & 0 \\
SDS 50 & 0.05 & 0.2 \\
CTAB 10 & 0 & 0 \\
CTAB 50 & 0.25 & 0.5 \\
\hline
\end{tabular}

loaded oil droplets. Calculation of the corresponding permeability based on Figure S5 shows lower oil permeability through K101-infused membrane compared to that from LLD analysis (see Table S3). This can be explained based on the fewer active (opened) pores in the emulsion separation experiments compared to the LLD experiments.

According to Table 3, at the low concentration of surfactants $(10 \% \mathrm{cmc})$, no water was observed in the permeate of both membranes. The applied TMP $(\leq 0.1$ bar) is lower than the LEP for the corresponding surfactant solutions through both liquid-infused membranes (see Figure 4b). During oil permeation experiments, the dry membrane becomes completely infused with hexadecane (see Supporting Information Figure S6b). The K101-infused membrane shows some regions of hexadecane with about $45 \%$ remaining infusion liquid (see Supporting Information Figure S6c and section "LLD analysis of hexadecane through liquid-infused membrane").

At a higher concentration of surfactants $(50 \% \mathrm{cmc})$, water was observed in the permeate from both membranes (see Supporting Information Figures S8 and S9c,d for pictures of the permeates). This observation was expected for the dry membrane because the applied TMP is slightly higher than the LEP of CTAB 50 (0.05 bar) and very close to that of SDS 50 $(0.15$ bar $)$ for a hexadecane-infused membrane. Because the TMP is lower than the LEP of the aforementioned surfactant solutions for K101-infused membranes (Figure 4b), no water was expected in the permeate. The LEP values were measured when pure surfactant solutions were pushed through K101infused membranes. However, in the oil permeation experiments, hexadecane forms a layer on the K101-infused membrane (Figure 6a). Therefore, the surfactant solution is in contact with a hexadecane layer rather than with $\mathrm{K} 101$, resulting in a lower IFT compared to K101 only (Figure 4a).

The presence of three liquids leads to two interfaces that have their corresponding IFT, that is surfactant solutionhexadecane $\left(\gamma_{12}\right)$ and hexadecane-K101 $\left(\gamma_{23}\right)$ (see Figure 6a). This leads to a higher combined LEP by the addition of IFTs, that is surfactant solution-hexadecane-K101 $\left(\gamma_{12}+\gamma_{23}\right)$ compared to that of the dry pore without liquid-lining, that is surfactant solution-hexadecane $\left(\gamma_{12}\right)$ (Figure $6 \mathrm{~b}$ ). By considering the IFT value between $\mathrm{K} 101-$ hexadecane $\left[\gamma_{23}=8.7 \pm\right.$ $0.08 \mathrm{mN} / \mathrm{m}$ (see Supporting Information Table S1)], the LEP for SDS and CTAB solutions with concentration of $50 \% \mathrm{cmc}$ would increase to 0.26 and 0.15 bar respectively, resulting in less water permeation.

Contact Angle Results. In order to have a better understanding regarding the membrane surface properties, contact angle measurements have been performed on dry and K101-infused membranes before and after oil permeation. The results of static as well as advancing receding contact angle (ARCA) measurements performed on dry and K101-infused membranes before and after oil permeation from $\mathrm{O} / \mathrm{W}$ emulsions containing SDS are shown in Table 4.

As can be seen in Table 4, K101-infused membranes still display slippery behavior after oil permeation indicated by the low contact angle hysteresis. The larger hysteresis compared to the fresh K101-infused membrane reveals likely some reduced K101 coverage on the membrane. The dry membrane showed a decrease in hysteresis upon oil permeation due to the infusion of hexadecane in the membrane after oil permeation (see Supporting Information Figure S5b).

A decrease in the water contact angle (static as well as dynamic, $\theta_{\mathrm{Adv}}$ and $\theta_{\mathrm{Rec}}$ ) is observed after oil permeation for both membranes, which can be explained by surfactant adsorption on the membrane surface.

\section{SUMMARY AND CONCLUSIONS}

In this work, we have reported on the permeation of oil droplets from oil-in-water $(\mathrm{O} / \mathrm{W})$ emulsions, containing watersoluble surfactants through liquid-infused and non-infused (dry) PVDF membranes. A fluorinated oil, PFPE (Krytox 101), has been used to fabricate liquid-infused membranes (K101-infused). To permeate a hexadecane oil phase, deadend filtration has been conducted. Four $\mathrm{O} / \mathrm{W}$ emulsions were made by dispersing $5 \mathrm{wt} \%$ hexadecane in surfactant solutions containing 10 and $50 \% \mathrm{cmc}$ of two water-soluble surfactants (SDS and CTAB). Based on the LEP of the surfactant solutions and hexadecane through dry and K101-infused membranes, a TMP smaller than 0.1 bar is sufficient for selective oil permeation. The results revealed that both membranes could selectively permeate oil and retain water from the $\mathrm{O} / \mathrm{W}$ emulsions containing a low concentration of surfactants $(10 \% \mathrm{cmc})$. At high surfactant concentrations $(50 \%$ $\mathrm{cmc}$ ) water was also observed in the permeate from both membranes. The amount of permeated water through a dry membrane was greater than that through a K101-infused membrane using the same surfactant. This can be explained by the presence of two interfaces, that is, surfactant-hexadecane

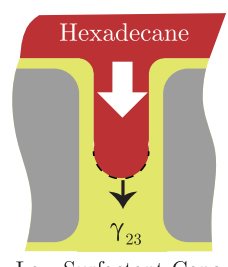

Low Surfactant Conc

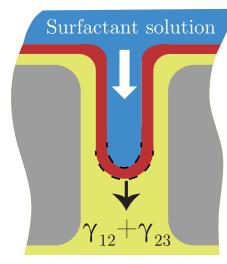

High Surfactant Conc.

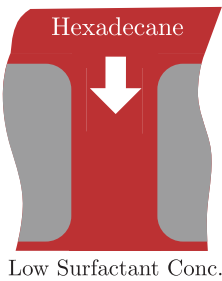

Low Surfactant Conc

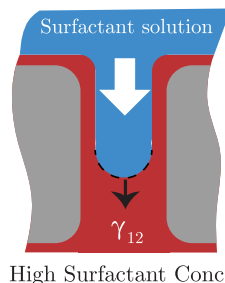

Figure 6. Schematic illustration of the interfaces in (a) K101-infused pore and (b) dry pore at low and high concentrations of surfactants. Yellow color shows K101 infusion liquid ( $\gamma_{12}$ and $\gamma_{23}$ are the IFT between surfactant solution-hexadecane and hexadecane-K101, respectively). 
Table 4. Static and ARCA Measurements on Dry and K101-Infused Membranes before and after Oil Separation from Emulsions Containing SDS (SDS 10 and SDS 50)

\begin{tabular}{|c|c|c|c|c|}
\hline \multirow[b]{2}{*}{ sample } & \multicolumn{4}{|c|}{ contact angle (deg) } \\
\hline & mean static & advancing $\left(\theta_{\text {Adv }}\right)$ & receding $\left(\theta_{\mathrm{Rec}}\right)$ & hysteresis \\
\hline K101-infused (before) & $117 \pm 0.1$ & $119 \pm 0.1$ & $114 \pm 0.3$ & $5.1 \pm 0.3$ \\
\hline K101-infused (SDS 10) & $102.81 \pm 1.98$ & $104.12 \pm 0.35$ & $93.90 \pm 0.51$ & $10.21 \pm 0.26$ \\
\hline K101-infused (SDS 50) & $102.42 \pm 0.65$ & $94.47 \pm 0.11$ & $91.46 \pm 0.39$ & $8.02 \pm 0.45$ \\
\hline dry (before) & $136 \pm 0.3$ & $142.3 \pm 0.26$ & $117 \pm 1.2$ & $25 \pm 1$ \\
\hline dry (SDS 10) & $103 \pm 0.68$ & $103.15 \pm 0.26$ & $88.25 \pm 0.51$ & $14.90 \pm 0.30$ \\
\hline dry (SDS 50) & $103.21 \pm 0.43$ & $98.32 \pm 0.14$ & $87.68 \pm 1.69$ & $10.64 \pm 1.60$ \\
\hline
\end{tabular}

$\left(\gamma_{12}\right)$ and hexadecane-K101 $\left(\gamma_{23}\right)$. The combined IFT $\left(\gamma_{12}+\right.$ $\left.\gamma_{23}\right)$, and thus the Laplace pressure, will be higher compared to that for a dry membrane with only the IFT of $\gamma_{12}$. This leads to an increase in the required LEP of the surfactant solution in case of the K101-infused membrane. This work demonstrates the potential of liquid-infused membranes for emulsion separations. The separation mechanism is governed by the corresponding IFTs between liquid phases involved. Much lower fluxes are required in case of selective permeation of the dispersed phase, providing routes to energy-efficient and low fouling membrane separation processes.

\section{ASSOCIATED CONTENT}

\section{S Supporting Information}

The Supporting Information is available free of charge on the ACS Publications website at DOI: 10.1021/acs.langmuir.9b01055.

Membrane characterization, physical properties of surfactant solutions and oils, pictures of fabricated emulsions, LLD analysis of hexadecane through liquidinfused membrane, permeate pictures of different $\mathrm{O} / \mathrm{W}$ emulsions from dry and liquid-infused membranes, experimental filtration results, droplet size distributions, and surfactant adsorption (PDF)

\section{AUTHOR INFORMATION}

\section{Corresponding Author}

*E-mail: r.g.h.lammertink@utwente.nl. Phone: +31 (0) 534892063.

\section{ORCID}

Rob G. H. Lammertink: 0000-0002-0827-2946

Notes

The authors declare no competing financial interest.

\section{ACKNOWLEDGMENTS}

This work is part of the Vici project STW 016.160.312 which is financed by the Netherlands Organisation for Scientific Research (NWO). This work was performed in the cooperation framework of Wetsus, European Centre of Excellence for Sustainable Water Technology (www.wetsus. nl). Wetsus is co-funded by the Dutch Ministry of Economic Affairs and Ministry of Infrastructure and Environment, the European Union Regional Development Fund, the Province of Fryslân and the Northern Netherlands Provinces.

\section{REFERENCES}

(1) Shannon, M. A.; Bohn, P. W.; Elimelech, M.; Georgiadis, J. G.; Mariñas, B. J.; Mayes, A. M. Science and technology for water purification in the coming decades. Nature 2008, 452, 301-310.
(2) Anderson, G. K.; Saw, C. B.; Le, M. S. Oil/Water separation with surface modified membranes. Environ. Technol. Lett. 1987, 8, 121132.

(3) Standards for Discharge of Effluent into Water or on Land; S. I. No. 5; National Environment Management Authority: Uganda, 1999.

(4) Padaki, M.; Surya Murali, R.; Abdullah, M. S.; Misdan, N.; Moslehyani, A.; Kassim, M. A.; Hilal, N.; Ismail, A. F. Membrane technology enhancement in oil-water separation. A review. Desalination 2015, 357, 197-207.

(5) Nandi, B. K.; Moparthi, A.; Uppaluri, R.; Purkait, M. K. Treatment of oily wastewater using low cost ceramic membrane: Comparative assessment of pore blocking and artificial neural network models. Chem. Eng. Res. Des. 2010, 88, 881-892.

(6) Chakrabarty, B.; Ghoshal, A. K.; Purkait, M. K. Ultrafiltration of stable oil-in-water emulsion by polysulfone membrane. J. Membr. Sci. 2008, 325, 427-437.

(7) Hlavacek, M. Break-up of oil-in-water emulsions induced by permeation through a microfiltration membrane. J. Membr. Sci. 1995, 102, 1-7, Engineering of Membrane Processes II Environmental Applications .

(8) Sherony, D. F.; Kintner, R. C.; Wasan, D. T. Coalescence of secondary emulsions in fibrous beds. Surf. Colloid Sci. 1978, 10, 99161.

(9) Mohammadi, T.; Kazemimoghadam, M.; Saadabadi, M. Modeling of membrane fouling and flux decline in reverse osmosis during separation of oil in water emulsions. Desalination 2003, 157, 369-375, Desalination and the Environment: Fresh Water for all .

(10) Zhong, J.; Sun, X.; Wang, C. Treatment of oily wastewater produced from refinery processes using flocculation and ceramic membrane filtration. Sep. Purif. Technol. 2003, 32, 93-98, Seventh International Conference on Inorganic Membranes .

(11) Ohya, H.; Kim, J. J.; Chinen, A.; Aihara, M.; Semenova, S. I.; Negishi, Y.; Mori, O.; Yasuda, M. Effects of pore size on separation mechanisms of microfiltration of oily water, using porous glass tubular membrane. J. Membr. Sci. 1998, 145, 1-14.

(12) Gryta, M.; Karakulski, K. The application of membrane distillation for the concentration of oil-water emulsions. Desalination 1999, 121, 23-29.

(13) Karakulski, K.; Kozlowski, A.; Morawski, A. W. Purification of oily wastewater by ultrafiltration. Sep. Technol. 1995, 5, 197-205.

(14) He, Y.; Jiang, Z.-W. Technology review: Treating oilfield wastewater. Filtr. Sep. 2008, 45, 14-16.

(15) Bodzek, M.; Konieczny, K. The use of ultrafiltration membranes made of various polymers in the treatment of oilemulsion wastewaters. Waste Manage. 1992, 12, 75-84.

(16) Chen, W.; Peng, J.; Su, Y.; Zheng, L.; Wang, L.; Jiang, Z. Separation of oil/water emulsion using Pluronic F127 modified polyethersulfone ultrafiltration membranes. Sep. Purif. Technol. 2009, 66, 591-597.

(17) Lin, Y.-M.; Rutledge, G. C. Separation of oil-in-water emulsions stabilized by different types of surfactants using electrospun fiber membranes. J. Membr. Sci. 2018, 563, 247-258.

(18) Zhang, J.; Xue, Q.; Pan, X.; Jin, Y.; Lu, W.; Ding, D.; Guo, Q. Graphene oxide/polyacrylonitrile fiber hierarchical-structured membrane for ultra-fast microfiltration of oil-water emulsion. Chem. Eng. J. 2017, 307, 643-649. 
(19) Wang, J.; Hou, L. a.; Yan, K.; Zhang, L.; Yu, Q. J. Polydopamine nanocluster decorated electrospun nanofibrous membrane for separation of oil/water emulsions. J. Membr. Sci. 2018, 547, $156-162$.

(20) Ge, J.; Zhang, J.; Wang, F.; Li, Z.; Yu, J.; Ding, B. Superhydrophilic and underwater superoleophobic nanofibrous membrane with hierarchical structured skin for effective oil-in-water emulsion separation. J. Mater. Chem. A 2017, 5, 497-502.

(21) Liao, Y.; Tian, M.; Wang, R. A high-performance and robust membrane with switchable super-wettability for oil/water separation under ultralow pressure. J. Membr. Sci. 2017, 543, 123-132.

(22) Yang, C.; Han, N.; Wang, W.; Zhang, W.; Han, C.; Cui, Z.; Zhang, X. Fabrication of a PPS Microporous Membrane for Efficient Water-in-Oil Emulsion Separation. Langmuir 2018, 34, 1058010590 .

(23) Xiang, Y.; Wang, Y.; Lin, H.; Wang, Y.; Xiong, Z.; Liu, F. Efficient separation of $\mathrm{O} / \mathrm{W}$ and $\mathrm{W} / \mathrm{O}$ micro-emulsion by thermally responsive superantiwetting PVDF membrane. React. Funct. Polym. 2015, 97, 86-95.

(24) Tao, M.; Xue, L.; Liu, F.; Jiang, L. An Intelligent Superwetting PVDF Membrane Showing Switchable Transport Performance for Oil/Water Separation. Adv. Mater. 2014, 26, 2943-2948.

(25) Pan, J.; Xiao, C.; Huang, Q.; Liu, H.; Zhang, T. ECTFE hybrid porous membrane with hierarchical micro/nano-structural surface for efficient oil/water separation. J. Membr. Sci. 2017, 524, 623-630.

(26) Chu, Z.; Feng, Y.; Seeger, S. Oil/Water Separation with Selective Superantiwetting/Superwetting Surface Materials. Angew. Chem., Int. Ed. 2015, 54, 2328-2338.

(27) Huang, M.; Si, Y.; Tang, X.; Zhu, Z.; Ding, B.; Liu, L.; Zheng, G.; Luo, W.; Yu, J. Gravity driven separation of emulsified oil-water mixtures utilizing in situ polymerized superhydrophobic and superoleophilic nanofibrous membranes. J. Mater. Chem. A 2013, 1, 14071-14074.

(28) Yang, H.-C.; Hou, J.; Chen, V.; Xu, Z.-K. Janus Membranes: Exploring Duality for Advanced Separation. Angew. Chem., Int. Ed. 2016, 55, 13398-13407.

(29) Wang, Z.; Wang, Y.; Liu, G. Rapid and Efficient Separation of Oil from Oil-in-Water Emulsions Using a Janus Cotton Fabric. Angew. Chem., Int. Ed. 2016, 55, 1291-1294.

(30) Turco, A.; Malitesta, C.; Barillaro, G.; Greco, A.; Maffezzoli, A.; Mazzotta, E. A magnetic and highly reusable macroporous superhydrophobic/superoleophilic PDMS/MWNT nanocomposite for oil sorption from water. J. Mater. Chem. A 2015, 3, 17685-17696.

(31) Abdulhussein, A. T.; Kannarpady, G. K.; Biris, A. S. One-step synthesis of a steel-polymer wool for oil-water separation and absorption. npj Clean Water 2019, 2, 10.

(32) Lefferts, A. G. Membranes as separators of dispersed emulsion phases. Ph.D. Thesis, Wageningen University, 1997.

(33) Wong, T.-S.; Kang, S. H.; Tang, S. K. Y.; Smythe, E. J.; Hatton, B. D.; Grinthal, A.; Aizenberg, J. Bioinspired self-repairing slippery surfaces with pressure-stable omniphobicity. Nature 2011, 477, 443447.

(34) Epstein, A. K.; Wong, T.-S.; Belisle, R. A.; Boggs, E. M.; Aizenberg, J. Liquid-infused structured surfaces with exceptional antibiofouling performance. Proc. Natl. Acad. Sci. U.S.A. 2012, 109, 13182-13187.

(35) Kim, P.; Wong, T.-S.; Alvarenga, J.; Kreder, M. J.; AdornoMartinez, W. E.; Aizenberg, J. Liquid-Infused Nanostructured Surfaces with Extreme Anti-Ice and Anti-Frost Performance. ACS Nano 2012, 6, 6569-6577.

(36) Hou, X.; Hu, Y.; Grinthal, A.; Khan, M.; Aizenberg, J. Liquidbased gating mechanism with tunable multiphase selectivity and antifouling behaviour. Nature 2015, 519, 70-73.

(37) Stone, H. A. Ice-Phobic Surfaces That Are Wet. ACS Nano 2012, 6, 6536-6540.

(38) Anand, S.; Paxson, A. T.; Dhiman, R.; Smith, J. D.; Varanasi, K. K. Enhanced Condensation on Lubricant-Impregnated Nanotextured Surfaces. ACS Nano 2012, 6, 10122-10129.
(39) Okada, I.; Shiratori, S. High-Transparency, Self-Standable GelSLIPS Fabricated by a Facile Nanoscale Phase Separation. ACS Appl. Mater. Interfaces 2014, 6, 1502-1508.

(40) Bazyar, H.; Javadpour, S.; Lammertink, R. G. H. On the Gating Mechanism of Slippery Liquid Infused Porous Membranes. Adv. Mater. Interfaces 2016, 3, 1600025.

(41) Hu, H.; Wang, J.; Wang, Y.; Gee, E.; Liu, G. Silicone-Infused Antismudge Nanocoatings. ACS Appl. Mater. Interfaces 2017, 9, 9029-9037.

(42) Hou, X. Smart Gating Multi-Scale Pore/Channel-Based Membranes. Adv. Mater. 2016, 28, 7049-7064.

(43) Laplace, P. S. Traité de mécanique céleste, tome quatriéme (Treatise on Celestial Mechanics, tome 4), Supplement au livre $X d u$ Traité de mécanique céleste. Sur l'action capillaire (Supplement to Book 10 of the Treatise on Celestial Mechanics. On the capillary action), P. 349-417; Courcier: Paris, France, 1805.

(44) Bazyar, H.; Lv, P.; Wood, J. A.; Porada, S.; Lohse, D.; Lammertink, R. G. H. Liquid-liquid displacement in slippery liquidinfused membranes (SLIMs). Soft Matter 2018, 14, 1780-1788.

(45) von Szyszkowski, B. Experimentelle Studien über kapillare Eigenschaften der wässerigen Lösungen von Fettsäuren. (Experimental studies of the capillary properties of aqueous solutions of fatty acids). Z. Phys. Chem. 1908, 64, 385-414.

(46) Lamb, S. H. Hydrodynamics, 6th ed.; Cambridge University Press: Cambridge University: United Kingdom, 1945. 